Bull. Austral. Math. Soc.

20D10, 20D99

Vol. 44 (1991) [19-31]

\title{
BOUNDS ON THE FITTING LENGTH OF FINITE SOLUBLE GROUPS WITH SUPERSOLUBLE SYLOW NORMALISERS
}

\author{
R.A. Bryce, V. Fedri and L. Serena
}

We prove that, in a finite soluble group, all of whose Sylow normalisers are supersoluble, the Fitting length is at most $2 m+2$, where $p^{m}$ is the highest power of the smallest prime $p$ dividing $\left|G / G^{\mathcal{S}}\right|:$ here $G^{\mathcal{S}}$ is the supersoluble residual of $G$. The bound $2 m+2$ is best possible. However under certain structural constraints on $G / G^{s}$, typical of the small examples one makes by way of experimentation, the bound is sharply reduced. More precisely let $p$ be the smallest, and $r$ the largest, prime dividing the order of a group $G$ in the class under consideration. If a Sylow p-subgroup of $G / G^{\mathcal{S}}$ acts faithfully on every $r$-chief factor of $G / G^{\mathcal{S}}$, then $G$ has Fitting length at most 3 .

\section{INTRODUCTION}

We denote by $N^{\mathcal{S}}$ the class of finite groups in which the normalisers of all Sylow subgroups are supersoluble. The motivation for the study of the class $N^{S}$ is a result of Bianchi et al. [1] which says that only nilpotent groups, among all finite groups, have nilpotent Sylow normalisers.

The study of soluble groups in $N^{\mathcal{S}}$ was begun in [3]. Several of the results proved there indicate a close connection between the structure of a soluble group $G$ in $N^{S}$ and that of its supersoluble co-radical $\bar{G}=G / G^{S}$. The aim of the present paper is to investigate this connection more closely. More precisely we investigate bounds on the Fitting length of a soluble group in $N^{\mathcal{S}}$ in terms of the structure of $\bar{G}$. While there are groups $G$ in $N^{S}$ of arbitrarily large Fitting length with given isomorphism type of $\bar{G}$ (see Theorem 3.3 of $[3]$, for example) we show that such groups necessarily involve just two primes in their order. For soluble groups in $N^{S}$ involving at least three primes we show that Fitting length is bounded by a linear function of a certain invariant.

THEOREM 1.1. Let $G$ be a soluble group in $N^{S}$ involving at least three distinct primes in its order. Let $p^{m}$ be the highest power of the smallest prime $p$ dividing $|\bar{G}|$. Then the Fitting length of $G$ is at most $2 m+2$, and this bound is best possible.

Other more technical results show that under certain restrictions on $\bar{G}$ this bound can be sharply reduced (see Theorem 4.1).

Received 10 July 1990

Much of this work was done while the last two named authors were visiting the Australian National University in Canberra. They express their appreciation for the warm hospitality shown to them. They also acknowledge with appreciation grants from M.P.I. and C.N.R. respectively.

Copyright Clearance Centre, Inc. Serial-fee code: 0004-9729/91 \$A2.00+0.00. 


\section{STATEMENT OF PRELIMiNaRY RESUltS AND NOTATIONS}

All groups treated in this article will be finite, and usually they are soluble as well. Most notation is standard. We list some here that may not be so standard.

- $\mathfrak{R}, \mathcal{S}$ and $\mathfrak{N}^{i}$ denote respectively the saturated formations of soluble groups, supersoluble groups and groups of Fitting length $i$.

- $G^{\mathfrak{A}}$ denotes the $\mathfrak{A}$-residual of the group $G$, where $\mathfrak{A}$ is a formation.

- $\bar{G}$ is the supersoluble co-radical $G / G^{\mathcal{S}}$ for a group $G$.

- $\pi(G)$ denotes the set of different primes dividing $|G|$.

- $\ell(G)$ is the Fitting length of the group $G$.

The results which follow will be quoted in the sequel. Some are given with reference, but no proof. Others are so easy or so well-known as to require no proof here.

Result 2.1. ([5, Lemma 1.6 of Chapter X]) Let $P$ be a Sylow subgroup of a $p$-soluble group $G$. Then $O_{p^{\prime}}\left(N_{G}(P)\right) \leqslant O_{p^{\prime}}(G)$.

REsult 2.2. If $r$ is the largest prime dividing the order of a supersoluble group $G$ then $G$ has a normal Sylow $r$-subgroup.

RESULT 2.3. If $S$ is a Sylow subgroup of $G$ and $M$ is a normal subgroup of $G$ then

$$
N_{G / M}(S M / M)=N_{G}(S) M / M \text {. }
$$

RESUlt 2.4. Let $G \in N^{\mathcal{S}} \cap \Re$ and let $M$ be a normal subgroup of $G$.

(a) If $M$ is minimal normal in $G$ then either $G$ is supersoluble or $\pi(G)=$ $\pi(G / M)$.

(b) If $\pi(G) \neq \pi(G / M)$ then $G / M$ is supersoluble.

Proof: In case (a) $M$ is a $p$-group for some prime $p$. If $G$ is not supersoluble then $M$ is not a Sylow $p$-subgroup of $G$. Hence $p \in \pi(G / M)$ and therefore $\pi(G)=\pi(G / M)$.

In case (b) let $1=M_{0}<M_{1}<\ldots<M_{n}=M$ be part of a chief series of $G$. Let $i$ be minimal with respect to the property $\pi(G) \neq \pi\left(G / M_{i}\right)$. Note that $0<i \leqslant n$. By (2.6) below and (a), $G / M_{i-1}$ is supersoluble; and therefore so is $G / M$.

RESULT 2.5. Let $G \in N^{\mathcal{S}}$ be a group of $p$-length 1 for some prime $p$. Then $G^{\mathcal{S}} \leqslant$ $O_{p^{\prime}}(G)$.

Proof: If $P$ is a Sylow $p$-subgroup of $G$, then $P O_{p^{\prime}}(G) / O_{p^{\prime}}(G)$ is normal in $G / O_{p^{\prime}}(G)$. However

$$
\begin{aligned}
G / O_{p^{\prime}}(G) & =N_{G / O_{p^{\prime}}(G)}\left(P O_{p^{\prime}}(G) / O_{p^{\prime}}(G)\right) \\
& =N_{G}(P) O_{p^{\prime}}(G) / O_{p^{\prime}}(G) \\
& \cong N_{G}(P) / N_{G}(P) \cap O_{p^{\prime}}(G)
\end{aligned}
$$

which is supersoluble since $G \in N^{\mathcal{S}}$. Hence $G^{\mathcal{S}} \leqslant O_{p^{\prime}}(G)$ as required. 
RESUlT 2.6. ([3, Lemma 1.1]) $N^{\mathcal{S}}$ is closed for factor groups.

Result 2.7. ([3, Lemma 4.5]) Let $G \in N^{\mathcal{S}}$. Then $\pi(G)=\pi(G)$

RESUlt 2.8. ([3, Lemma 4.7]) Let $G \in N^{\mathcal{S}} \cap \mathfrak{R}$ and suppose that $p, q \in \pi(G)$ with $p<q$. If $\bar{P}, \bar{Q}$ are respectively a Sylow $p$-subgroup and a Sylow $q$-subgroup of $\bar{G}$ with $\bar{Q} \leqslant N_{\bar{G}}(\bar{P})$, then $G$ has $p$-length 1 .

RESUlt 2.9. ([3, Lemma 4.9]) Let $G \in N^{S} \cap \Re$. If $p, q, r \in \pi(G)$ with $p<q<r$ and if $q, r \in \pi\left(G / O_{p^{\prime}}(G)\right)$ then $O_{q^{\prime}}(G) \neq 1$.

Result 2.10. Let $G \in N^{S} \cap \Re$. Then $G$ is nilpotent if and only if $\bar{G}$ is nilpotent.

ProOF: One direction is easy. So suppose that $\bar{G}$ is nilpotent. Let $r$ be the largest prime dividing $|G|$. By (2.5) and (2.8), $G^{\mathcal{S}} \leqslant O_{p^{\prime}}(G)$ for all primes $p \neq r$. Hence $G^{\mathcal{S}}$ is an $r$-group. Then (2.2) ensures that $G$ has a normal Sylow $r$-subgroup, and so $G$ is supersoluble. Therefore $G^{\mathcal{S}}=1$ and so $G$ is nilpotent.

REsult 2.11. Let $G \in N^{\mathcal{S}} \cap \Re$. Then $G$ is metanilpotent if and only if it is supersoluble.

Proof: Again one direction is easy. Suppose therefore that $G$ is metanilpotent. By way of obtaining a contradiction, suppose that $G^{\mathcal{S}} \neq 1$, and that $G$ is minimal with this property. Every proper factor group of $G$ is then supersoluble. It follows that $G$ has a unique minimal normal subgroup $M=G^{\mathcal{S}}$ which is complemented: here we use the fact that the class of supersoluble groups is saturated. Now $M=F(G)$ so, since $G$ is metanilpotent, $\bar{G}=G / M$ is nilpotent. By (2.10) therefore $G$ is nilpotent, a contradiction. Hence $G$ is supersoluble.

One feature of this proof is worth highlighting for later quotation.

ReSUlt 2.12. A group $G \in N^{\mathcal{S}} \cap \Re$, minimal with respect to not belonging to a given saturated formation, is primitive (meaning here that $G$ has a complemented unique minimal normal subgroup).

REsult 2.13. Let $G=M H$ where $M$ is a nilpotent normal subgroup of $G$. Then if $G \in N^{\mathcal{S}}$ so is $H \in N^{\mathcal{S}}$.

ProOf: Let $P$ be a Sylow p-subgroup of $H$ and $M_{0}$ a Sylow $p$-subgroup of $M$. Then $P M_{0}$ is a Sylow $p$-subgroup of $G$; and clearly $N_{H}(P) \leqslant N_{G}\left(P M_{0}\right)$, and therefore it is supersoluble. Hence $H \in N^{\mathcal{S}}$.

Result 2.14. Let $G$ be a group in which, for some prime $r, O_{r}(G)$ is cyclic and $O_{r^{\prime}}(G)=1$; and let $H$ be a complement for $O_{r}(G)$ in $G$. If $M$ is a faithful irreducible module for $G$ over a field, then $M_{H}$ is a direct sum of regular modules. 
Proof: Write $R=O_{r}(G)$. Let $k$ be the ground field of $M$. We may suppose that $k$ is algebraically closed since, if $k^{*}$ is the closure of $k$, then $k^{*} \otimes M$ is a direct sum of faithful irreducible modules for $G$ over $k^{*}$ ([2, 70.15]). If each of these irreducible summands is a direct sum of regular modules for $H$, then so is $k^{*} \otimes M$ and therefore so is $M$. So suppose that $k$ is closed. Let $M_{1}$ be an irreducible component of $M_{R}$ with inertia subgroup $U$. Then $R=U$; for, if not, we have some $h \in H \cap U, h \neq 1$ and $[h, R] \neq 1$. Therefore $M_{1} \not M_{1} h$ since $R$ acts by scalars on $M$. It follows that $R=U$ and hence that $M=M_{1}^{G}$ whence $M_{H}$ is a direct sum of regular modules.

\section{PROOF OF THEOREM 1.1}

Let $\mathcal{A}$ be a sub-class of $N^{\mathcal{S}} \cap \Re$ with the following properties:

(i) for all $G \in \mathcal{A}$ either $|\pi(G)| \geqslant 3$ or $G$ is supersoluble;

(ii) $\boldsymbol{Q} \mathcal{A}=\mathcal{A}$;

(iii) whenever $H$ is a Hall $\{p, q, r\}$-subgroup of an $\mathcal{A}$-group $G$ where $p, q, r$ are distinct primes, respectively the smallest, second largest and largest in $\pi(G)$, for which $H^{\mathcal{S}}=H \cap G^{\mathcal{S}}$ then $H \in \mathcal{A}$.

Theorem 3.1. Let $\mathcal{A}$ satisfy the properties listed above, and let $d$ be an integer greater than or equal to 3. Suppose that $\mathcal{A} \not \mathcal{N}^{d}$ and let $G$ have minimal order with respect to $G \in \mathcal{A} \backslash \mathcal{N}^{d}$.

Then

(a) $G$ is a primitive group;

(b) $|\pi(G)|=3$;

and if $\pi(G)=\{p, q, r\}$, where $p<q<r$, and $T$ is a $H$ all $\{q, r\}$-subgroup of $G$ and $P$ a Sylow $p$-subgroup of $G$, then

(c) $G=P T$ and $T \unlhd G$;

(d) $G^{\mathcal{S}}=T^{\mathcal{S}}$;

(e) $G^{S} / G^{\mathfrak{n}^{3}}$ is a q-group.

Proof: By (2.12) $G$ is a primitive group so (a) is satisfied. We write $M$ for the unique minimal normal subgroup of $G$, and $H$ for a complement for $M$ in $G$. Note that $M$ is a $q$-group for some prime $q$. It will be convenient to denote by $p, r$ the smallest and largest members respectively of $\pi(G)$. Denote

$$
\bar{\pi}=\{t \in \pi(G): t>q\}
$$

Of course $\bar{\pi}$ may be empty. One goal on the way to a proof of (3.1) is to prove

$$
|\bar{\pi}| \leqslant 1
$$


Let $Q$ be a Sylow $q$-subgroup of $G$ and consider $N_{G}(Q)$. By $(2.1) O_{q^{\prime}}\left(N_{G}(Q)\right) \leqslant$ $O_{g^{\prime}}(G)=1$, so $Q$ is the Fitting subgroup of $N_{G}(Q)$. Since $N_{G}(Q)$ is supersoluble, $N_{G}(Q)$ is a $\bar{\pi}^{\prime}$-group. Also $N_{G}(Q) / Q$ is abelian. By (2.3) we see that

$$
N_{G / G^{s}}\left(Q G^{s} / G^{\mathcal{S}}\right)
$$

is also a $\bar{\pi}^{\prime}$-group. Since $G / G^{S}=\bar{G}$ is supersoluble it follows that $O_{\bar{\pi}}(G)$ is a nilpotent (Hall) $\bar{\pi}$-subgroup of $\bar{G}$. (To see this suppose that $s, t \in \bar{\pi}$ with $s<t$. If $S, T, \bar{Q}$ are respectively Sylow $s$-, $t$ - and $q$-subgroups of $\bar{G}$, we can choose them so that $\bar{Q}$ normalises $S$. Then $[\bar{Q}, S]$ centralises every $t$-chief factor of $\bar{G}$ since $\bar{G}$ is supersoluble, and therefore centralises $T$. But under the condition that $N_{\bar{G}}(Q)$ is a $\bar{\pi}$-group we have that $[\bar{Q}, S]=S$. That is $[S, T]=1$. It follows that $O_{\bar{\pi}}(\bar{G})$ is nilpotent.) Now by (2.5) and (2.8) we have that

$$
G^{\mathcal{S}} \leqslant O_{t^{\prime}}(G), \quad t \text { prime }, \quad q<t<r .
$$

On the other hand if $t$ is a prime satisfying $t \in \pi(G), t<q<r$, then since $O_{q^{\prime}}(G)=1$ we conclude from (2.9) that $O_{t^{\prime}}(G)$ contains a Sylow subgroup $U$ of $G$ (either a Sylow $q-$, or a Sylow $r-$, subgroup). By the Frattini argument therefore $G=N_{G}(U) O_{t^{\prime}}(G)$ whence $G / O_{t^{\prime}}(G)$ is supersoluble since $N_{G}(U)$ is. We deduce that

$$
G^{\mathcal{S}} \leqslant O_{t^{\prime}}(G), \quad t \text { prime, } \quad t<q<r .
$$

It then follows from (3.3) and (3.4) that

$$
\text { either } q=r \text { or } G^{\mathcal{S}} \text { is a }\{q, r\} \text {-group. }
$$

In the first case $\bar{\pi}$ is empty, so (3.2) certainly holds. Consider the case $q \neq r$, and suppose, in contradiction to (3.2), that $|\bar{\pi}| \geqslant 2$. We claim first that $G^{\mathcal{S}} / G^{\mathfrak{N}^{3}}$ is a $q$-group. For, let $X / G^{\Re^{3}}=O_{q}\left(G^{s} / G^{\Re^{3}}\right)$. Then $G / X$ has normal Sylow $r$-subgroup and so $G / X$ is supersoluble, whence

$$
G^{\mathcal{S}} \leqslant X \leqslant G^{\mathcal{S}}
$$

so $G^{\mathcal{S}}=X$, as required.

Now $G^{\mathfrak{n}^{3}} \neq 1$ since $d \geqslant 3$. Let $G^{\mathfrak{N}^{3}} / Y$ be a chief factor of $G$; it is an $r$-chief factor since $G^{\mathcal{S}}$ is a $\{q, r\}$-group and otherwise $G^{\mathfrak{n}^{3}} \leqslant Y$. Let $Z$ be a normal subgroup of $G$ containing $Y$ and maximal with respect to not containing $G^{n^{3}}$. Then $G / Z$ has nilpotent length 4 exactly and $G^{\mathfrak{N}^{3}} Z / Z$ is its unique minimal normal subgroup. 
Let $G_{0}=G / Z$. By $(2.4), \pi\left(G_{0}\right)=\pi(G)$ and therefore $\pi(G)=\pi\left(G_{0}\right)$. Now $O_{r^{\prime}}\left(G_{0}\right)=1$ so, by (2.1), if $R_{0}$ is a Sylow $r$-subgroup of $G_{0}$ then $R_{0}$ is the Fitting subgroup of $N_{G_{0}}\left(R_{0}\right)$. Therefore $N_{G_{0}}\left(R_{0}\right) / R_{0}$ is abelain.

Also

so

$$
\begin{aligned}
G_{0} & =G_{0}^{S} N_{G_{0}}\left(R_{0}\right) \\
G_{0} / R_{0} G_{0}^{S} & =N_{G_{0}}\left(R_{0}\right) R_{0} G_{0}^{S} / R_{0} G_{0}^{S} \\
& \cong N_{G_{0}}\left(R_{0}\right) / R_{0}\left(N_{G_{0}}\left(R_{0}\right) \cap G_{0}^{\mathcal{S}}\right)
\end{aligned}
$$

which is abelian.

However the assumption that $|\bar{\pi}| \geqslant 2$ and the fact that $\bar{\pi}(G)=\pi\left(G_{0}\right)$ means that there is a prime $s$ satisfying $q<s<r$ and there are commuting $q$ - and $s$ - Sylow subgroups of $\bar{G}_{0}$. By (2.8) and (2.5) therefore

$$
G_{0}^{\mathcal{S}} \leqslant O_{q^{\prime}}\left(G_{0}\right)
$$

However

$$
\begin{aligned}
& G_{0}^{s} / G_{0}^{\Re^{3}}=\left(G^{s} Z / Z\right) / G^{\Re^{3}} Z / Z \\
& \cong G^{s} Z / G^{\Re^{3}} Z \\
& \cong G^{\mathcal{S}} G^{\mathfrak{N}^{3}} Z / G^{\mathfrak{\Re}^{3}} Z \\
& \cong G^{\mathcal{S}} / G^{\mathcal{S}} \cap G^{\mathfrak{N}^{3}} Z \\
& =G^{s} / G^{n^{3}}\left(G^{s} \cap Z\right)
\end{aligned}
$$

which is a $q$-group. Being also a $q^{\prime}$-group it is 1 . That is

$$
G_{0}^{\mathfrak{N}^{2}} \leqslant G_{0}^{S} \leqslant G_{0}^{\mathfrak{N}^{3}}
$$

whence $G_{0}^{\mathfrak{n}^{2}}=1$, a contradiction to the assumption $|\bar{\pi}| \geqslant 2$.

When $q \neq r$ we have shown above that $G^{S}$ is a $\{q, r\}$-group and that $q$ is the second largest prime in $\pi(G)$. In the case $q=r$ we have a similar result in that $G^{S}$ still involves only two primes, $r$ and the next largest. To see this let us re-define $q$ in this case to be the second largest prime in $\pi(G)$; since we shall not need again in this section the particular prime dividing $|M|$ this will not cause confusion. Let $R$ be a Sylow $r$-subgroup of $G$. Then the argument above involving $G / Z$ shows that $G / R G^{S}$ is abelian (we can formally set $Z=1$ in the penultimate paragraph). Hence (2.8) and (2.5) show that

$$
G^{\mathcal{S}} \leqslant O_{t^{\prime}}(G)
$$

for every prime $t<q$. Therefore $G^{S}$ is again a $\{q, r\}$-group. To sum up we have in all cases: 
Result 3.6. Let $q, r$ be the two largest primes dividing $|G|$. Then $G^{\mathcal{S}}$ is a $\{q, r\}$ group.

The proof of (e) of Theorem 3.1 now follows as in the paragraph following (3.5).

Finally let $T$ be a Hall $\{q, r\}$-subgroup of $G$. Then $G^{\mathcal{S}} \leqslant T \unlhd G$. Moreover if $R$ is a Sylow $r$-subgroup of $G$ then $T^{\mathcal{S}} R \unlhd G$ and therefore $G / T^{\mathcal{S}}$ is supersoluble. Hence $G^{\mathcal{S}} \leqslant T^{\mathcal{S}} \leqslant G^{\mathcal{S}}$ so $G^{\mathcal{S}}=T^{\mathcal{S}}$, as required by (d). Let $P$ be a Sylow p-subgroup of $G$ and put $L=P T$. Then $L^{\mathcal{S}}=G^{\mathcal{S}}$ and $L$ is a Hall subgroup of $G$, so $L \in \mathcal{A}$. Moreover $L$ and $G$ have the same Fitting length since by (2.11), $L^{\mathfrak{N}^{2}}=G^{\mathfrak{N}^{2}}$. If $L \neq G$ the minimality of $G$ means that $L \in \mathcal{N}^{d}$ and therefore $G \in \mathcal{N}^{d}$, a contradiction. Hence $L=G$. This completes the proof of (b) and with it the proof of the theorem.

We are now in a position to establish Theorem 1.1

ProOF OF ThEOREM 1.1: Let $m$ be a positive integer. Let $\mathcal{A}$ be the subclass of $N^{S} \cap \Re$ of all groups $G$ for which either $G$ is supersoluble, or $|\pi(G)| \geqslant 3$ and if $p^{t}$ is the largest power of the smallest prime dividing $|\bar{G}|$, then $t \leqslant m$. Let $d=2 m+2$ and, on the assumption that $\mathcal{A} \ \mathcal{N}^{d}$, choose a minimal group $G$ in $\mathcal{A} \backslash \mathcal{N}^{d}$.

We observe that this class $\mathcal{A}$ satisfies the properties postulated at the start of this section. Hence $G$ has the structure described in Theorem 3.1. We now invoke the Kurzweil result [6] to conclude that

$$
\begin{aligned}
\ell(G)=\ell(T) & \leqslant \ell\left(C_{T}(P)\right)+2 m \\
& \leqslant 2 m+2
\end{aligned}
$$

since $C_{T}(P) \leqslant N_{G}(P)$ which is supersoluble. This contradiction concludes the proof of (1.1), apart from giving an example to show that $2 m+2$ is the best possible bound.

Let $p, q, r$ be primes with $p|q-1, p| r-1$ and $q \mid r-1$. For a group $H$ in which $O_{q}(H)=1$ we define a group $\widehat{H}$ as follows. Let $P$ be cyclic of order $p$ and $M$ a faithful irreducible module for $P \times H$ over $\mathbb{F}_{q}$. Then let $N$ be faithful and irreducible for the semi-direct product $M(P \times H)$ over $\mathbb{F}_{r}$. Denote by $\widehat{H}$ the semi-direct product $N M(P \times H)$. Of course $\widehat{H}$ is not uniquely determined by this description, but any of the groups which satisfy it will do for $\widehat{H}$.

Now let $H_{1}$ be the Frobenius group of order $q r$. If $H_{i}$ has been defined for $i \geqslant 1$ define

$$
H_{i+1}=\widehat{H}_{i}
$$

We leave it as an exercise for the reader to check that for all $m \geqslant 1, H_{m} \in N^{s} \cap \Re$, $\left|\bar{H}_{m}\right|$ is divisible by $p^{m}$ and by no larger power of $p$, and $\ell\left(H_{m}\right)=2 m+2$. Therefore the bound on Fitting length determined in the proof of Theorem 1.1 is best possible. $\square$ 


\section{A particular bound}

In the example just given the $p$ - and $r$-Sylow subgroups of the supersoluble coradical centralise one another. The aim of this section is to show that groups in $N^{\mathcal{S}} \cap \mathfrak{R}$ which satisfy an extreme opposite of this condition have very much more rigidly bounded Fitting length.

THEOREM 4.1. Let $G \in N^{\mathcal{S}} \cap \mathfrak{R}$ with $|\pi(G)| \geqslant 3$ and suppose that in $\bar{G}$ a Sylow $p$-subgroup of $\bar{G}$ acts faithfully on every $r$-chief factor of $\bar{G}$ : here $p, r$ are respectively the smallest and largest primes dividing $|G|$.

Then $\ell(G) \leqslant 3$.

The proof begins by defining the following sub-class $\mathcal{A}_{0}$ of $N^{\mathcal{S}} \cap \mathfrak{R}: G \in \mathcal{A}_{0}$ if and only if either $G$ is supersoluble, or $|\pi(G)| \geqslant 3$ and $\bar{G}$ has the property described in the statement of the theorem, that is if $p, r$ are respectively the smallest and largest primes dividing $|G|$ then a Sylow $p$-subgroup of $\bar{G}$ acts faithfully on every $r$-chief factor of $\bar{G}$. Observe that $\mathcal{A}_{0}$ satisfies the properties (i), (ii), (iii) at the head of Section 3.

Now on the assumption that the result is false we choose a minimal group $G$ in $\mathcal{A}_{0} \backslash \mathcal{N}^{3}$. Then Theorem 3.1 yields a description of its structure, and we use the notation of that theorem in the sequel without further comment. However some more notation will be necessary. $G$ has a unique minimal normal subgroup $M$ with complement $\boldsymbol{H}$. Set $K=H^{\mathcal{S}}$ and note that $K$ is a $q$-group and $M$ an $r$-group.

We begin now a lengthy further reduction of the structure of $G$, ending eventually with a proof that it cannot, in fact, exist. This reduction will be broken up into a number of steps.

RESULT 4.2. $K$ has nilpotency class at most 2 and $K / K^{\prime}$ is a chief factor of $H$.

The following lemma will be useful in proving this.

LEMMA 4.3. Let $K_{0}$ be a non-trivial normal subgroup of $H$ properly contained in $K$. If $R$ is a Sylow $r$-subgroup of $H$ then $\left[K_{0}, R\right]=1$.

Proof: Choose a supplement $S$ of $K$ in $H$ as follows. Since $K / \Phi(K)$ is abelian and $K / \Phi(K)=(H / \Phi(K))^{\mathcal{N}^{2}}$ there is by [4, Lemma] a complement $S_{0} / \Phi(K)$ for $K / \Phi(K)$ in $H / \Phi(K)$. Choose $S$ to be a minimal supplement for $K$ in $H$ with $S \leqslant S_{0}$. This means that $S \cap K \leqslant \Phi(S)$, since otherwise a proper subgroup of $S$ would do as well: and therefore $S$ is supersoluble because $S / S \cap K \cong S K / K$ is supersoluble, and the class of supersoluble groups is saturated. Note that $K_{0} S \neq H$ since otherwise $K=K_{0}(K \cap S)=K_{0}, K \cap S$ being Frattini in $K$.

Define $G_{0}=M K_{0} S$. We prove that $G_{0} \in N^{\mathcal{S}}$. Since $G_{0}$ contains a Sylow $p$ subgroup and a Sylow $r$-subgroup of $G$ it suffices to show that $N_{G_{0}}\left(Q_{0}\right)$ is supersoluble 
whenever $Q_{0}$ is a Sylow $q$-subgroup of $G_{0}$. Now

$$
\left[N_{G_{0}}\left(Q_{0}\right) \cap M, Q_{0}\right] \leqslant M \cap Q_{0}=1 \text {. }
$$

However this means in particular that $K_{0}$ centralises $N_{G_{0}}\left(Q_{0}\right) \cap M$, a contradiction unless $N_{G_{0}}\left(Q_{0}\right) \cap M=1$ (by Fitting's Lemma $M$ can have no non-trivial fixed points for $K_{0}$ ). consequently

$$
\begin{aligned}
N_{G_{0}}\left(Q_{0}\right) & \cong N_{G_{0}}\left(Q_{0}\right) / N_{G_{0}}\left(Q_{0}\right) \cap M \\
& \cong N_{G_{0}}\left(Q_{0}\right) M / M \\
& \cong N_{G_{0} / M}\left(Q_{0} M / M\right) \\
& \cong N_{K_{0} S}\left(Q_{0}\right)
\end{aligned}
$$

which is supersoluble by (2.13) because $H \in N^{\mathcal{S}}$. Therefore $G_{0} \in N^{\mathcal{S}}$.

We now suppose, contrary to the statement of Lemma 4.3, that $K_{1}=\left[K_{0}, R\right] \neq 1$ where we choose $R$ to be a Sylow $r$-subgroup of $S$, and therefore of $H$ : observe that $K_{1}$ is independent of the choice of this Sylow $r$-subgroup. Note that $K_{1} \unlhd K_{0} S$ and that $\left[M, K_{1}\right] \neq 1$.

A simple calculation shows that $G_{0}^{\mathcal{N}^{3}} \geqslant\left[M, K_{1}\right]>1$. However note that $C_{M}\left(K_{1}\right) \unlhd G_{0}$ and that

$$
M=C_{M}\left(K_{1}\right) \times\left[M, K_{1}\right] .
$$

If we define $G_{1}=G_{0} / C_{M}\left(K_{1}\right)$, it is in $\mathcal{A}_{0}$. It is in $N^{\mathcal{S}}$ because $G_{0}$ is; and

$$
\begin{aligned}
G_{1} / G_{1}^{\mathcal{S}} & \cong\left(G_{0} / C_{M}\left(K_{1}\right)\right) /\left(G_{0}^{\mathcal{S}} C_{M}\left(K_{1}\right) / C_{M}\left(K_{1}\right)\right) \\
& \cong G_{0} / G_{0}^{\mathcal{S}} C_{M}\left(K_{1}\right)
\end{aligned}
$$

which is a factor group of $G_{0} / M$ since $\left[M, K_{1}\right] \leqslant G_{0}^{\mathcal{N}^{3}} \leqslant G_{0}^{S}$. In $G_{0} / M$ a Sylow p-subgroup is represented faithfully on every $r$-chief factor. It follows that $G_{1} \in \mathcal{A}_{0}$.

By the minimality of $G, G_{1} \in \mathcal{N}^{3}$. Hence

$$
\left[M, K_{1}\right] \leqslant G_{0}^{\mathcal{N}^{3}} \leqslant C_{M}\left(K_{1}\right)
$$

a contradiction. We have proved therefore that $K_{1}=1$ and so the proof of Lemma 4.3 is complete.

One immediate consequence of Lemma 4.3 is that $K / K^{\prime}$ is a chief factor of $H$ and that $\left[K^{\prime}, R\right]=1$. To prove (4.2) we may as well suppose that $K^{\prime} \neq 1$. Suppose, contrary to the statement of (4.2), that $K$ has class $c \geqslant 3$. Consider the function $\theta: K / K^{\prime} \rightarrow \gamma_{c}(K)$ defined, for $y \in \gamma_{c-1}(K)$, by

$$
\left(x K^{\prime}\right) \theta=[x, y]
$$


This is a well-defined $R$-homomorphism into $\gamma_{c}(K)$. Now $K / K^{\prime}$ is an $R$-module with no non-trivial fixed points, and $\gamma_{c}(K)$ is a trivial $R$-module. Hence $[K, y]=\left(K / K^{\prime}\right) \theta=$ 1 , and this for every $y$ in $\gamma_{c-1}(K)$. However this means that $\gamma_{c}(K)=\left[K, \gamma_{c-1}(K)\right]=$ 1, a contradiction to $K$ having class $c$. Therefore $K$ has class 2, completing (4.2).

Next we show that

RESULT 4.4. $K^{\prime} \leqslant \zeta_{1}(H)$.

We may suppose $K^{\prime} \neq 1$. Let $X$ be a Hall $\{p, q\}$-subgroup of $S$. Then $M K^{\prime} X \leqslant$ $N_{G}(M R)$ which is supersoluble. Also $M=F\left(M K^{\prime} X\right)$ since $M$ is self-centralising. We deduce that $K^{\prime} X \cong M K^{\prime} X / M$ is abelian and therefore $\left[K^{\prime}, X\right]=1$. However $\left[K^{\prime}, R K\right]=1$ so $\left[K^{\prime}, H\right]=1$ as required by (4.4).

We now see at once that

Result 4.5. $K$ is extra special.

For, since $H$ is represented faithfully and irreducibly on $M, K^{\prime}$ cyclic. Also $\zeta_{1}(K)=K^{\prime}$ since $K / K^{\prime}$ is a chief factor, and we have assumed that $K^{\prime} \neq 1$. It follows that $K$ is extra-special.

This now leads to a proof that

RESUlt 4.6. $K$ is a minimal normal subgroup of $H$.

It suffices to show that $K^{\prime}=1$. On the assumption that it is not (4.5) yields that $\left|K^{\prime}\right|=q$. Now, by (2.14), $C_{K / K^{\prime}}(P) \neq 1$, and $C_{M}(P) \neq 1$, where $P$ is a Sylow $p$-subgroup of $X$, and therefore of $G$. Hence

$$
K^{\prime}<C_{K}(P)
$$

and

$$
C_{M}(P) C_{K}(P) \leqslant N_{G}(P)
$$

which is supersoluble. It follows that $C_{K}(P)$ is abelian. For, if not, $C_{K}(P)^{\prime}=K^{\prime}$ and so $\left[C_{M}(P), K^{\prime}\right]=1$, a contradiction since, by Fitting's Lemma, $K^{\prime}$ has no non-trivial fixed points in $M$. However $K=[K, P] C_{K}(P)$. Consider the function $\phi:[K, P] \rightarrow K^{\prime}$ defined, for $y \in C_{K}(P)$, by

$$
x \phi=[x, y] \text {. }
$$

This is a $P$-homomorphism, and therefore

$$
\begin{aligned}
{[K, P] \phi } & =[K, P, P] \phi \\
& \leqslant\left[K^{\prime}, P\right] \phi \\
& =1 .
\end{aligned}
$$

It follows that $C_{K}(P)$ centralises $[K, P]$, and hence that

$$
K^{\prime}<C_{K}(P) \leqslant \zeta_{1}(K)=K^{\prime}
$$


a contradiction. Therefore $K^{\prime}=1$, and the proof of (4.6) is complete.

Our next step is to show that

$$
|R|=r .
$$

This is accomplished by a rather shorter, but similar method to that which we used to reduce $K$. Let $R_{0}$ be a normal subgroup of $S$ properly contained in $R$. To prove (4.7) it suffices to show that $R_{0}=1$, and we use an indirect proof, assuming that it is not. Note that $\left[K, R_{0}\right]=K$ since, indeed, $C_{R}(K)=1$.

Consider the subgroup $G_{2}=M K R_{0} X$. We claim that $G_{2} \in N^{S}$. For this it suffices to show that $N_{G_{2}}\left(M R_{0}\right)$ is supersoluble. Note that $N_{G_{2}}\left(M R_{0}\right) \geqslant M X R_{0}$ and hence

Now

$$
N_{G_{2}}\left(M R_{0}\right)=M X R_{0}\left(K \cap N_{G_{2}}\left(M R_{0}\right)\right) \text {. }
$$

$$
\left[K \cap N_{G_{2}}\left(M R_{0}\right), R_{0}\right] \leqslant K \cap M R_{0}
$$$$
=1
$$

which means that $N_{G_{2}}\left(M R_{0}\right) \cap K=1$ since $R_{0}$ has no non-trivial fixed points in $K$. Therefore

$$
\begin{aligned}
N_{G_{2}}\left(M R_{0}\right) & =M X R_{0} \\
& \leqslant N_{G}(M R)
\end{aligned}
$$

which is supersoluble. Therefore $G_{2} \in N^{\mathcal{S}}$. Also $G_{2} \in \mathcal{A}_{0}$ because since

$$
M K \leqslant G_{2}^{\mathcal{N}^{2}} \leqslant G_{2}^{\mathcal{S}} \leqslant G^{\mathcal{S}}=M K
$$

so $G_{2} / G_{2}^{S}$ is isomorphic to a subgroup of $G / G^{S}$. By the minimality of $G, G_{2} \in \mathcal{N}^{3}$. However $G_{2}^{\mathcal{N}^{3}}=M$ which is a contradiction, so the assumption that $R_{0} \neq 1$ is wrong. This completes the proof of (4.7).

Our aim now is to examine the representation of $H$ on $M$. We need some structural notation to do this. Note that $K$ is complemented in $H$ since $K$ is abelian, and equal to $H^{\mathcal{N}^{2}}$ : here using the result [4]. Hence we may take $S$ to be a complement for $K$ in $H$, containing $R$. Also $R$ has an abelian $\{p, q\}$-complement $X$ in $S$.

Now let $M_{1}$ be an irreducible component of $M_{K}$, with kernel $D$, so that $|K: D|=$ $q$. Let $U$ be the inertia subgroup of $M_{1}$. Note that $U$ contains no $r$-elements since, were it to do so, $D$ would admit the action of a Sylow $r$-subgroup of $H$, and therefore $R$ would act on $K / D$, giving fixed points for $R$ in $K$. Since $U=K(U \cap S)$ we may choose $X$ so that $U \cap S \leqslant X$. Then $X$ normalises $U$, and we may regard $M$ as induced from a $U X$-module $M_{2}$ with $M_{1} \leqslant M_{2}$. That is $M=M_{2}^{H}$. 
Write $X=P Q_{0}$ where $P$ is a Sylow $p$-subgroup, and $Q_{0}$ a Sylow $q$-subgroup, of $X$. By (2.14), $K$ is a direct sum of regular modules for $X / C_{X}(R)=X / C_{Q_{0}}(R)$. Define $K_{0}=C_{K}(P)$. Then $K_{0}$ is a direct sum of regular modules for $Q_{0} / C_{Q_{0}}(R)$. Hence there is a non-trivial element $c_{0}$ in $\left(K_{0} Q_{0}\right)^{\prime}$ which we may choose to be centralised by $Q_{0}$. Of course $c_{0} \in K_{0}$. Since $C_{G}(P)$ contains $C_{M}(P) K_{0} Q_{0}$, which is therefore supersoluble, we have that $\left(K_{0} Q_{0}\right)^{\prime}$ centralises $C_{M}(P)$. In particular

$$
\left[c_{0}, C_{M}(P)\right]=1
$$

Write $P=\langle x\rangle$ and suppose $|P|=p^{m}$. For $1 \neq m_{0} \in M_{2}$ and $1 \neq y \in R$ the following element,

$$
\mu=\sum_{i=0}^{p^{m}-1} m_{0} x^{i} \otimes y^{x^{i}}
$$

is in $C_{M}(P)$ : the proof is left as an exercise for the reader. It follows from (4.8) that $\mu c_{0}=\mu$ and this yields that $m_{0}=m_{0} y c_{0} y^{-1}$. In other words

$$
c_{0}^{y} \in D \text { for all } y \in R, y \neq 1
$$

Let $B=\left\{c_{0}^{y}: y \in R\right\}$. Then $|B|=r$ since $c_{0}^{y}=c_{0}^{y^{\prime}}$ is equivalent to asking that $y^{\prime} y^{-1}$ centralise $c_{0}$ and this to $y=y^{\prime}$. Moreover $B$ admits the action of $H$ and so $\langle B\rangle \unlhd H$. This means that $\langle B\rangle=K$ since $K$ is minimal normal in $H$. Now $\operatorname{dim} K$ is a multiple of $\left|X / C_{X}(R)\right|$ which is composite, while $|B|$ is prime, so it follows that $B$ is linearly dependent. But from a non-trivial linear relation on $B$ we deduce that

$$
\begin{aligned}
c_{0} & \in\left\langle c_{0}^{y}: 1 \neq y \in R\right\rangle \\
& \subseteq D
\end{aligned}
$$

by (4.9). Therefore $K=\langle B\rangle \leqslant D$, a contradiction. The proof of Theorem 4.1 is therefore complete.

\section{REFERENCES}

[1] M.G. Bianchi, A. Gillio Berta Mauri and P. Hauck, 'On finite groups with nilpotent Sylow normalizers', Arch. Math. 47 (1986), 193-197.

[2] C.W. Curtis and I. Reiner, Representation theory of finite groups and associative algebras (Interscience, New York and London, 1962).

[3] V. Fedri and L. Serena, 'Finite soluble groups with supersoluble Sylow normalizers', Arch. Math. 50 (1988), 11-18.

[4] G. Higman, 'Complementation of abelian normal subgroups', Publ. Math. Debrecen 4 (1955), 455-458. 
[5] B. Huppert and N. Blackburn, Finite groups III: Grundlehren Math. Wiss. 242 (SpringerVerlag, Berlin, Heidelberg, New York, 1982).

[6] H. Kurzweil, 'p-Automorphismen von aufiösbaren p'-Gruppen', Math. Z. 120 (1971), 326-354.

Department of Mathematics

Faculty of Science

Australian National University

GPO Box 4

Canberra City ACT 2601

Australia
Istituto Matematico

Università degli Studi di Firenze

vis Morgagni, 67/A

50135 Firenze

Italy 